\title{
Exploration of Potential Molecular Targets of Dexmedetomidine in the Intestinal Repair of Burnt Rats
}

\author{
Chao Qin \\ Yi Jiang \\ Mingdong $\mathrm{Yu}$ \\ Yingxue Bian \\ Yonghao Yu (D) \\ 'Department of Anesthesiology, Tianjin \\ Medical University General Hospital, \\ Tianjin, 300052, People's Republic of \\ China; ${ }^{2}$ Tianjin Institute of \\ Anesthesiology, Tianjin, 300000, People's \\ Republic of China; ${ }^{3}$ State Key Laboratory \\ of Medicinal Chemical Biology (Nankai \\ University), Tianjin, 30007I, People's \\ Republic of China; ${ }^{4}$ Department of \\ Anesthesiology, Tianjin Union Medical \\ Center, Tianjin, 300I2I, People's \\ Republic of China
}

Correspondence: Yonghao Yu Department of Anesthesia, Tianjin Medical University General Hospital, No. 154 Anshan Road, Heping District, Tianjin, 300052, People's Republic of China

Tel +86-13920590099

Email yyu@tmu.edu.cn
Background: More and more burn survivors were suffering from varying degrees of damage to the intestinal barrier. Dexmedetomidine (Dex) was frequently used as sedative in more cases, but it was found to have repair effect on intestinal barrier dysfunction recently. This study aimed to explore the potential specific targets of Dex in intestinal barrier repair in burn rats model.

Methods: Male adult SD rats were used to establish 40\% TBSA III degree scald model in our study. The samples were divided into four groups: burn rats (Burn), burn rats with Dex medication (Burn-Dex), sham rats (Sham) and sham rats with Dex medication (Sham-Dex). And plasma FITC-dextran and diamine oxidase (DAO) were detected to determine the intestinal permeability. Differentially expressed proteins were further adopted to proteinprotein interaction network analysis, Gene Ontology analysis (GO) and Kyoto Encyclopedia of Genes and Genomes (KEGG) pathway analysis.

Results: It showed that 40\% TBSA III degree scald model was successfully constructed. And plasma FITC-dextran and DAO decreased significantly after Dex administration. Additionally, differentially expressed genes Psmb10, Psmb7 among the experimental groups were screened, which were significantly enriched in proteasome and other several pathways. Conclusion: The results above suggested that Q4KM35 and Q9JHW0, which are encoded by Psmb10 and Psmb7, respectively, are two possible protein targets of Dex in intestinal barrier repair.

Keywords: dexmedetomidine, intestinal barrier, Psmb10, Psmb7

\section{Introduction}

A recent report by the World Health Organization estimates that burns will cause 180,000 deaths each year. ${ }^{1,2}$ What is more worrying is that burn survivors often suffer from varying degrees of damage to the intestinal barrier, ${ }^{3}$ whose development is usually associated with complex factors including stress, ischemia, hypoxia, ischemia-reperfusion injury, proinflammatory cytokines, etc. ${ }^{3-6}$ As we all know, intestinal barrier could prevent bacteria and endotoxin from translocating to other distant organs. ${ }^{7-9}$ The burn patients with intestinal barrier dysfunction usually almost lose this function and are at higher risk for bacterial translocation, sepsis, and even mortality. ${ }^{10,11}$ Thus, it will be of great significance if we could find specific drugs and screen specific biomarkers for intestinal barrier repair in burnt patients. 
Dexmedetomidine (Dex) is frequently used as sedative and anxiolytic, and has increasingly been studied for potential analgesic properties. ${ }^{12,13}$ In clinical treatments, besides used for the postoperative period and ICUs, ${ }^{14}$ Dex has been also demonstrated to reduce inflammation and to have protective effects of ischemia/reperfusion injury. ${ }^{15}$ Regarding the Dex mechanisms of action, a research showed that the reducing inflammation in sepsis may be involved in the $\alpha 2 \mathrm{~A}$ adrenoceptors in the astrocytes by Dex treatment. ${ }^{16}$ In intestinal mucosa and muscles, Dex may attenuate the surgical stress and the microcirculatory blood flow intensity. ${ }^{17,18}$ As the research continues to expand, in some studies, researchers found that intestinal barrier function of burn injuries with Dex has been repaired to a certain extent compared with the one without Dex. ${ }^{19}$ Actually, in endotoxemic rats, Dex has been reported that it can reduce intestinal epithelial cell death and tight junction damage, which may contribute to septic patient therapy. ${ }^{19,20}$ A recent study found that Dex had positive anti-apoptotic and antiinflammatory effects in intestinal tissue. ${ }^{21}$ However, there is no further research about the possible molecular targets of Dex in the intestinal repair, which limits the application of Dex in the repair of intestinal barrier. Therefore, it is necessary to screen specific targets of Dex in intestinal repair.

Based on the above background, via physiological indicators and proteomics analysis, we here purposed to explore the potential specific targets of Dex and its underlying possible mechanisms in intestinal barrier repair in burn rats model.

\section{Materials and Methods Ethics Approval}

Our study has been approved by the Animal Ethical and Welfare of Tianjin Medical University (Tianjin, China) (ethic code: SYXK (Tianjin): 2019-0004). All animal experiments were conducted in accordance with the guidelines by the Chinese legislation on the ethical use and care of laboratory animals. In China the guidelines are called the Animal Management Regulations and the Guidelines on the Kind Treatment of Laboratory Animals ([2006] No. 398).

\section{Sample Information and Sprague-Dawley Model}

Healthy and clean male adult SD rats were used for our study, weighing 250-300 g, 8-12 weeks old. They were purchased from Huafukang Bioscience Company (licence number SCXK 2019-0008; Beijing, China).

After anesthesia with $10 \mathrm{~g} / \mathrm{L}$ pentobarbital sodium intraperitoneal injection $(40 \mathrm{mg} / \mathrm{kg})$, using a super temperature-controlled scald instrument, scald to cause injury on the rats back at $92^{\circ} \mathrm{C}$ for 18 s to establish $40 \%$ TBSA III degree scald model (confirmed by pathological section of skin tissue, called burn).

\section{Experimental Setting}

The samples were divided into four groups: burn rats (Burn), burn rats with Dex medication (Burn-Dex), sham rats (Sham) and sham rats with Dex medication (ShamDex), and there are 8 replicates in each group.

Rats in all groups were administered $0.9 \%$ normal saline $(2 \mathrm{~mL} / \mathrm{kg} / \mathrm{h})$ for $3 \mathrm{~h}$. Subsequently, rats in the ShamDex group and the Burn-Dex group were injected with Dex $(5 \mu \mathrm{g} / \mathrm{kg} / \mathrm{h})$ for $4 \mathrm{~h},{ }^{19,22}$ and rats in the Sham group and the Burn group were administered the identical dose of $0.9 \%$ normal saline by continuous infusion.

\section{Histological Analysis}

Intestine tissue about $1 \mathrm{~cm}$ from the cecum was harvested after withdrawal $6 \mathrm{~h}$. Subsequently, the sample was fixed, paraffin-embedded, sectioned, deparaffinized, dehydrated and stained with hematoxylin and eosin as the previous methods. ${ }^{23}$ Histological analysis of intestine was quantified by Chiu's score. ${ }^{24}$

\section{Measurement of Fluorescein Isothiocyanate (FITC) Concentration in Plasma}

Rats were anesthetized, whose ileum was ligated about $10 \mathrm{~cm}$ from the terminal, and then $1 \mathrm{~mL}$ fluoresceinlabeled glucose of isothiocyanate (FITC-dextran, 10mg/ $\mathrm{mL}$, Sigma, U.S) was injected into the intestinal lumen. After abdominal closure for $30 \mathrm{~min}$, abdominal aortic blood was extracted and the FITC-dextran was detected to evaluate the permeability of intestinal.

\section{Measurement of Diamine Oxidase (DAO) Concentration in Plasma}

Blood of rat was taken from the abdominal aorta after anesthesia, plasma was obtained by centrifugation at the condition of $4^{\circ} \mathrm{C}, 3500 \mathrm{~g}$ for $10 \mathrm{~min}$. Concentration of DAO was measured by Elisa kit (Wuhan Mskbio 
Bioengineering Institute, China,) accordance with the instructions provided by the manufacturer.

\section{Sample Preparation for LC-MS/MS}

The protein was extracted from the sample using SDT lysis solution (4\% (w/v) SDS, $100 \mathrm{mM}$ Tris- $\mathrm{HCl}(\mathrm{pH} 7.6)$, $0.1 \mathrm{M}$ DTT), and BCA method was used for protein quantification. Peptide fragment was obtained by Filter Aided Proteome Preparation (FASP) ${ }^{25}$ then desalted, lyophilized and redissolved in $40 \mu \mathrm{L} 0.1 \%$ formic acid solution.

\section{LC-MS/MS Analysis}

Samples were separated by Easy n-LC. Solution A was $0.1 \%$ formic acid aqueous solution, and Solution B was $0.1 \%$ formic acid acetonitrile aqueous solution ( $84 \%$ acetonitrile). The column was maintained with $95 \%$ solution A, and pretreated samples were automatically loaded to the loading column (Thermo Scientific Acclaim PepMap100, $100 \mu \mathrm{m}^{*} 2 \mathrm{~cm}$, nanoViper C18), and then separated by the analytical column (Thermo scientific EASY column, $10 \mathrm{~cm}$, ID $75 \mu \mathrm{m}, 3 \mu \mathrm{m}, \mathrm{C} 18-\mathrm{A} 2)$. The flow rate was $300 \mathrm{~nL} / \mathrm{min}$.

The samples were separated by chromatography and analyzed by Q-EXactive mass spectrometer. Quantification of peptides was identified by MaxQuant software (version 1.5.3.17).

\section{Differential Expression Protein Analysis}

Differential expression analysis was based on the limma ${ }^{26}$ function package of the $\mathrm{R}$ language (version 3.5.2, the same below), and use the absolute value of the logarithmic conversion differential expression multiple $\left(\log _{2} \mathrm{FC}\right)$ and FDR $\leq 0.05$ as the standard to screen differential expression proteins between Burn-Dex vs Burn, Burn vs Sham, Sham-Dex vs Sham.

\section{Protein-Protein Interaction (PPI) Network Analysis and Enrichment Analysis}

The STRING database is a database for analyzing and predicting protein functional connections and interactions. We use STRING ${ }^{27}$ (https://string-db.org/, version 11.0) to analyze the protein functional connections and protein interactions. Taking protein names as input files, the STRING database was also used to perform GO, KEGG, and Reactome enrichment analysis. When FDR $<0.05$, it is significantly enriched.

\section{Statistical Analysis}

Differences in each groups were performed by KruskalWallis rank-sum test, with $p<0.05$ as threshold. Statistical analysis was conducted by $\mathrm{R}$ software version 3.5.2. All data were presented as mean $\pm \mathrm{SD}$.

\section{Results Intestinal Injury Model Successfully Constructed}

As shown in HE-stained intestine tissues, intact villi without obvious abnormality in the rats of Sham and Sham-Dex group (Figure 1A and B), and no significant difference between them. However, the histopathologic results showed that intestine tissues were seriously damaged in Burn group, which was scattered bleeding, decrease of villi tissue and goblet cells, as well as inflammatory cells infiltration (Figure 1C). Moreover, compared with Burn group, the histological destructiveness was released in Burn-Dex group, inflammatory cells decreased, and intestinal tissue slightly damaged (Figure 1D). In addition, as shown in the Figure 1E and Table S1, the Chiu's score markedly increased in Burn (P $<0.001)$ and Burn-Dex group $(\mathrm{P}<0.001)$, meanwhile, the score was notably alleviated in the Burn-Dex group $(\mathrm{P}<0.001)$.

\section{Effect of Dex on Intestinal Repair in Burned Rats}

The intestinal permeability was determined level of FITCdextran concentration after withdrawal $6 \mathrm{~h}$, compared with Sham and Sham-Dex group, the FITC-dextran concentration of Burn $(\mathrm{P}<0.001)$ and Burn-Dex group $(\mathrm{P}<0.001)$ was significantly increased, while the level of FITCdextran in Sham and Sham-Dex group basically the same $(\mathrm{P}>0.05)$. Besides, the FITC-dextran in Burn was obviously higher than Burn-Dex $(\mathrm{P}<0.001)$ (Figure 2A and Table S2).

$\mathrm{DAO}$ was a functional enzyme in intestines. The trend of DAO level in each group was similar to that of FITC-dextran in each group. There was no significant difference in DAO concentration $(\mathrm{P}>0.05)$. After withdrawal 6h, DAO in Burn $(\mathrm{P}<0.001)$ and Burn-Dex group $(\mathrm{P}<0.001)$ was distinctly higher than that in Sham and Sham-Dex group (Figure 2B and Table S2). These results revealed that the DEX could reduce the intestinal barrier dysfunction after burn injury. 
A

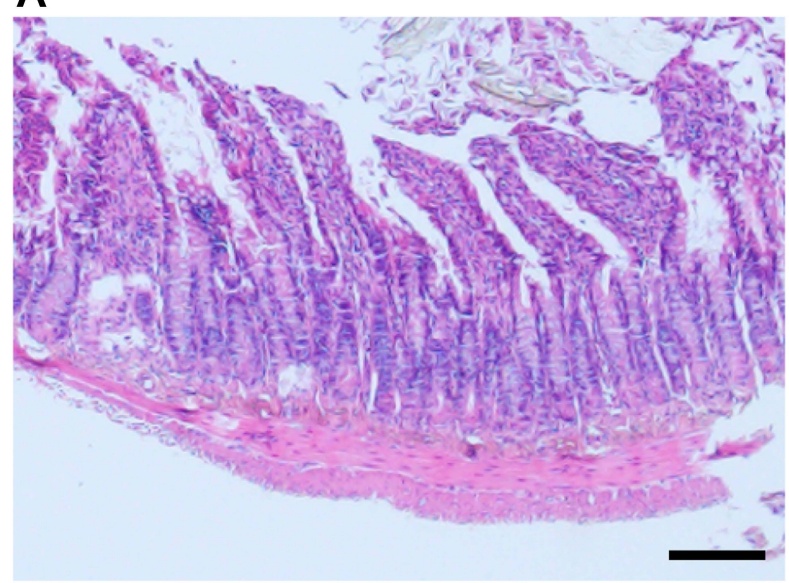

C

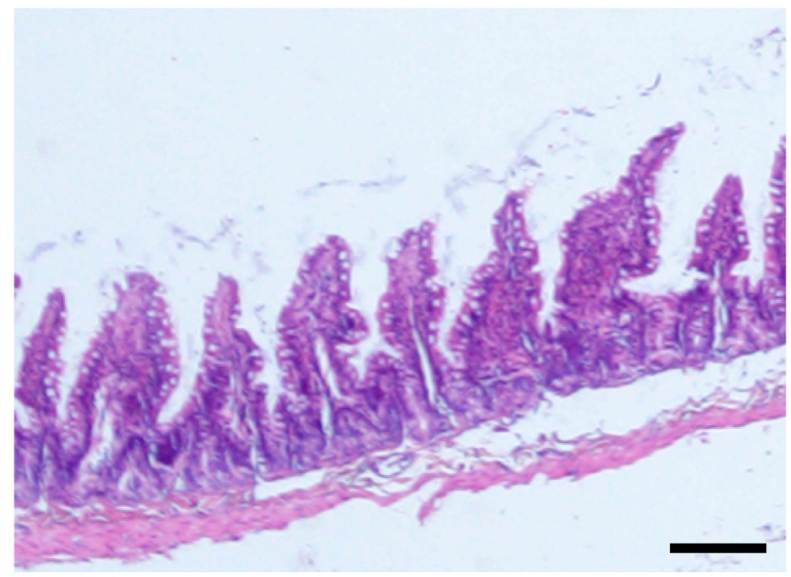

E

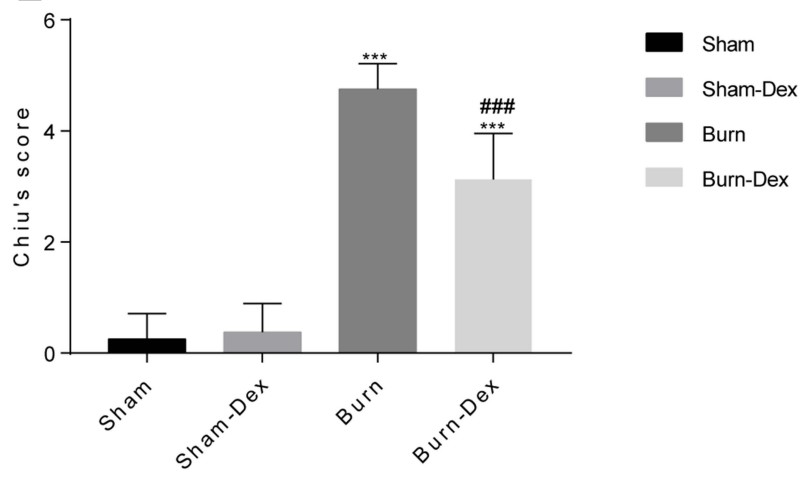

B

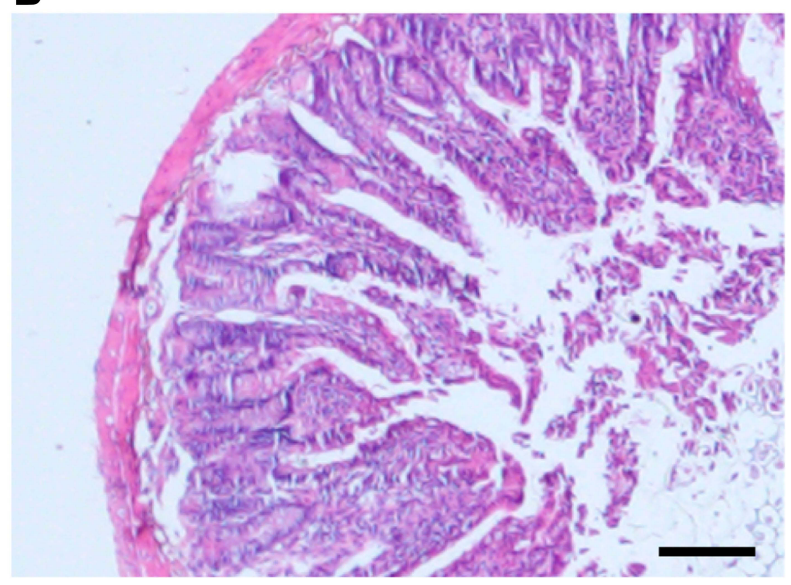

D

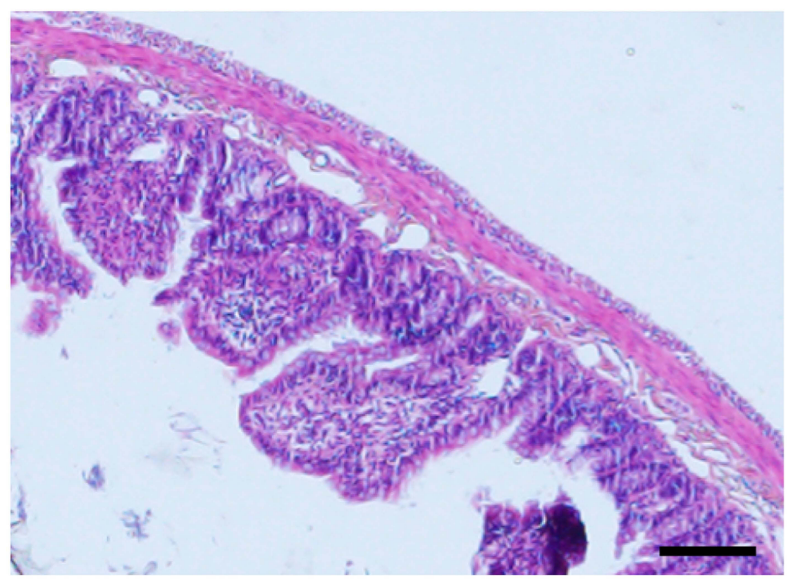

Figure I Histological examination of distal ileum. (A) Sham group. (B) Sham-Dex group. (C) Burn group. (D) Burn-Dex group. (E) Intestinal Chiu's score of each group. $* * * \mathrm{P}<0.001$, vs Sham group. ${ }^{1} \mathrm{P}<0.001$, vs Burn group. Scale bar $=100 \mu \mathrm{m}$.

\section{Differential Expression Proteins}

Besides the detection of the above physiological indicators, then we further analyzed the differentially expressed proteins in various experimental groups. Compared with Burn, there are 7 differentially expressed proteins in BurnDex, including 3 up-regulated proteins and 4 down- regulated proteins (Figure 3A). The expression levels of the differentially expressed proteins are significantly different between groups (Figure 3B). There are 13 differentially expressed proteins between the Burn and the Sham, including 10 up-regulated proteins and 3 down-regulated proteins (Figure 3C). The expression levels of the 
A

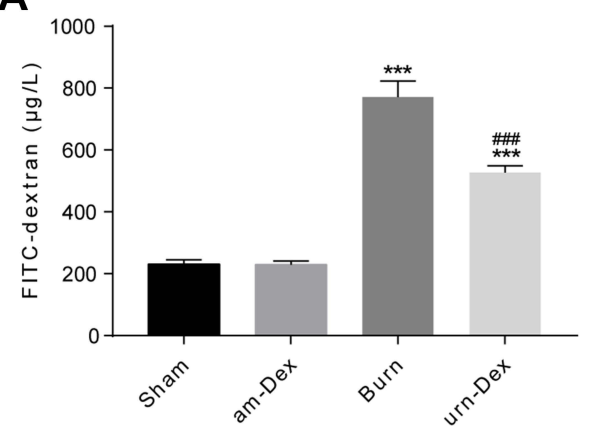

B

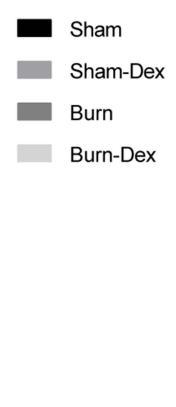

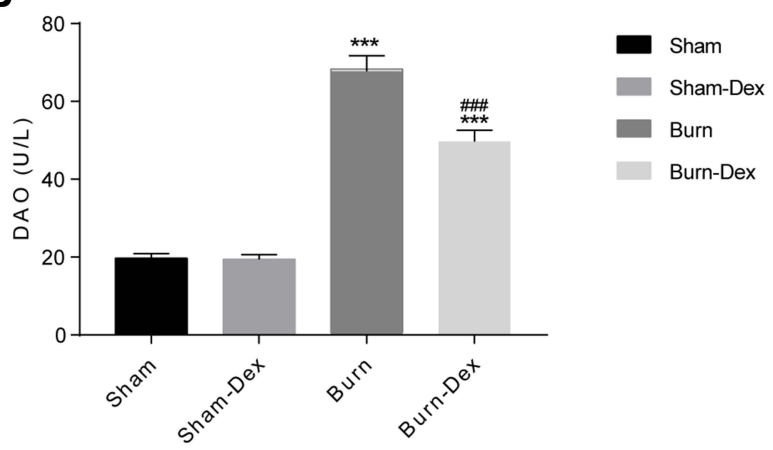

Figure 2 Plasma FITC-dextran and DAO concentration after withdrawal 6h. (A) FITC-dextran. (B) DAO. $* * * P<0.00$ I, vs Sham group. ${ }^{\# \#} \mathrm{P}<0.00$, vs Burn group.

differentially expressed proteins are significantly different between groups (Figure 3D). There are 7 differentially expressed proteins between the Sham-Dex and the Sham, including 6 up-regulated proteins and 1 down-regulated protein (Figure 3E). The expression levels of differentially expressed proteins were significantly different between groups (Figure 3F).

\section{Results of Function Enrichment Analysis}

We believe that proteins meet the following conditions may be the possible targets of Dex: a.) differentially express in Burn-Dex and Burn, b.) differentially express in Burn and Sham, c.) do not differentially express in Sham-Dex and Sham. There are 4 proteins that satisfied all of the above conditions, they are Q4KM35, Q9JHW0, A0A0G2K896 and A0A0H2UHM7, the corresponding rat GeneSymbols are respectively Psmb10, Psmb7, RGD1310507 and LOC100909441.

To further understand the potential biological functions of the differentially expressed proteins and the possible pathways they participate in, we annotated them using GO and KEGG database. They were significantly enriched in the three GO terms: proteolysis involved in cellular protein catabolic process, proteasome core complex and threonine-type endopeptidase activity (Table 1). Meanwhile, they were also significantly enriched in 49 pathways such as Proteasome, among which the most significant top 10 pathways are shown in Table 2 , and the detailed results are shown in Table S3.

\section{PPI Network and Screening Key Genes}

In order to find the key genes more accurately, we used the STRING database to construct a PPI network for these four proteins (Q4KM35, Q9JHW0, A0A0G2K896 and A0A0H2UHM7), then screened interaction pairs with the minimum required interaction score $>0.4$ as the threshold. The PPI network is shown in Figure S1. In Figure S1, the node represents a gene, and the edge represents the interaction between them. As shown in Figure S1, we find that there is interaction between Psmb10 and Psmb7.

\section{Discussion}

In this study, we mainly explored the repair effect of Dex and Dex's possible targets in intestinal barrier dysfunction after burns in rats. On the one hand, we detected that plasma FITC-dextran (fluorescein isothiocyanate-dextran) and plasma DAO (diamine oxidase) decreased significantly after Dex administration. On the other hand, we also screened differentially expressed proteins Psmb10, Psmb7 among the experimental groups, which were significantly enriched in Proteasome pathway.

Numerous animal and clinical studies have documented that the intestinal barrier was often broken down by severe burn injury, ${ }^{5,28}$ which was usually devastating blow for patients. Dexmedetomidine (Dex), besides used as sedative and anxiolytic, ${ }^{12}$ was explored in intestinal barrier repair. ${ }^{29}$ However, to the best of our knowledge, no previous study focused on the targets of Dex in intestinal barrier repair. We have found the possible targets of Dex in intestinal barrier repair, which should contribute to the understanding of its intestinal barrier repair function for burnt patients.

Firstly, we demonstrated that Dex indeed reduced intestinal permeability and repaired intestinal barrier in rats. It has been documented that impairments in the intestinal barrier manifest as intestinal permeability increasing. ${ }^{30-32}$ When the permeability of the intestine is abnormally increased, the DAO and FITC-dextran in the lumen will pass through the intestinal mucosa easily and go into the peripheral blood. ${ }^{33-36}$ Thus, they are now 
A

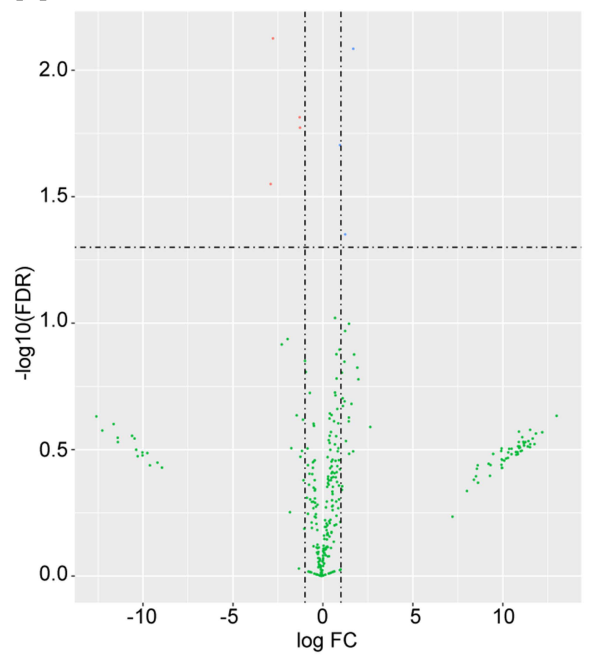

C

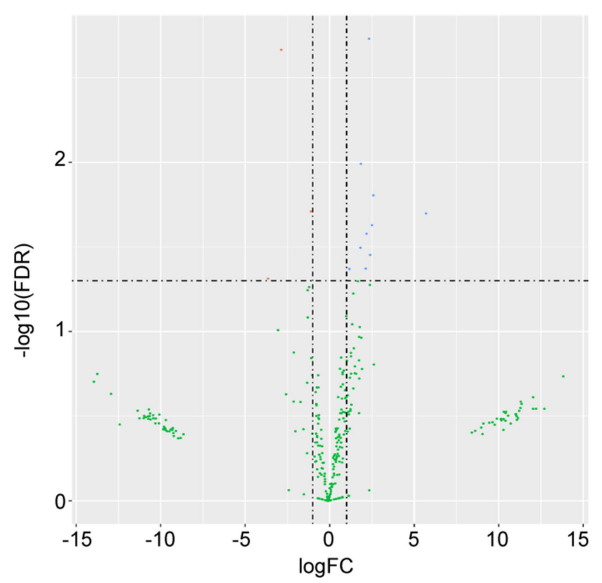

E

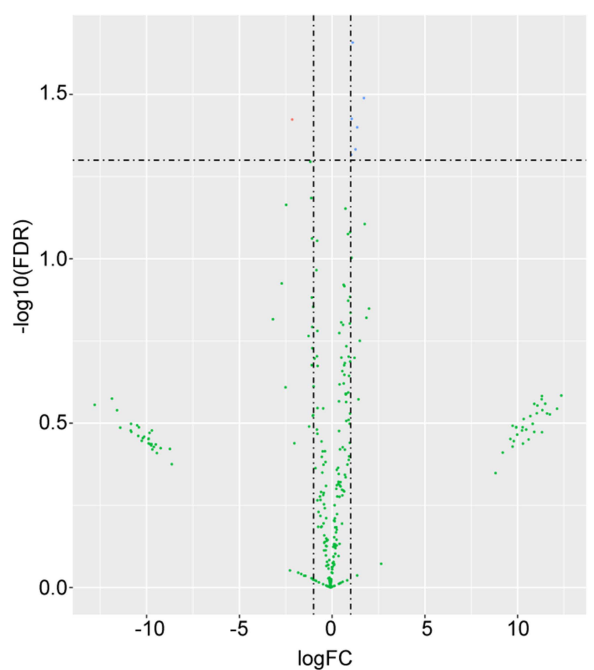

B

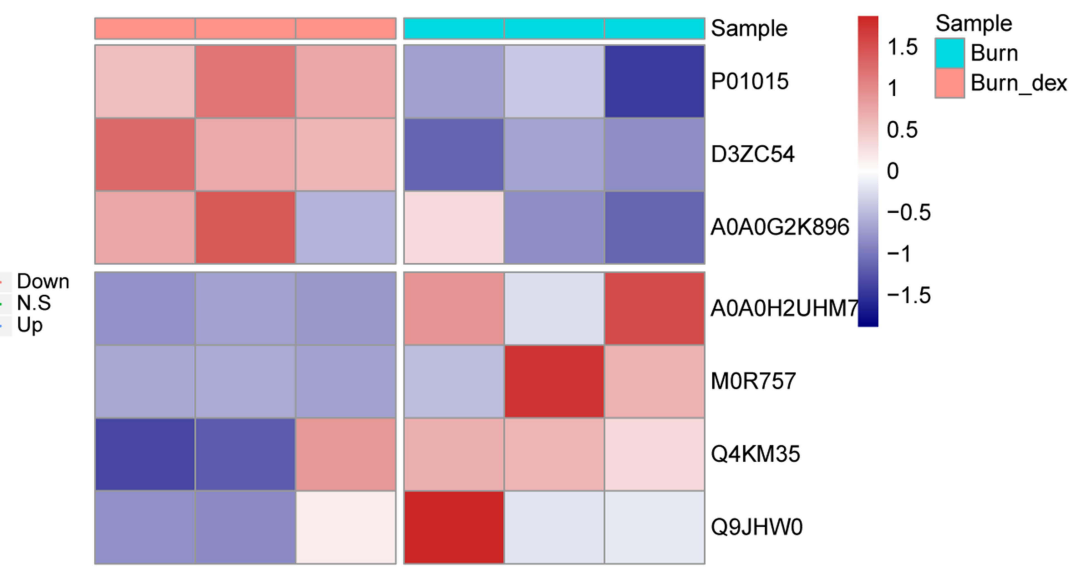

D

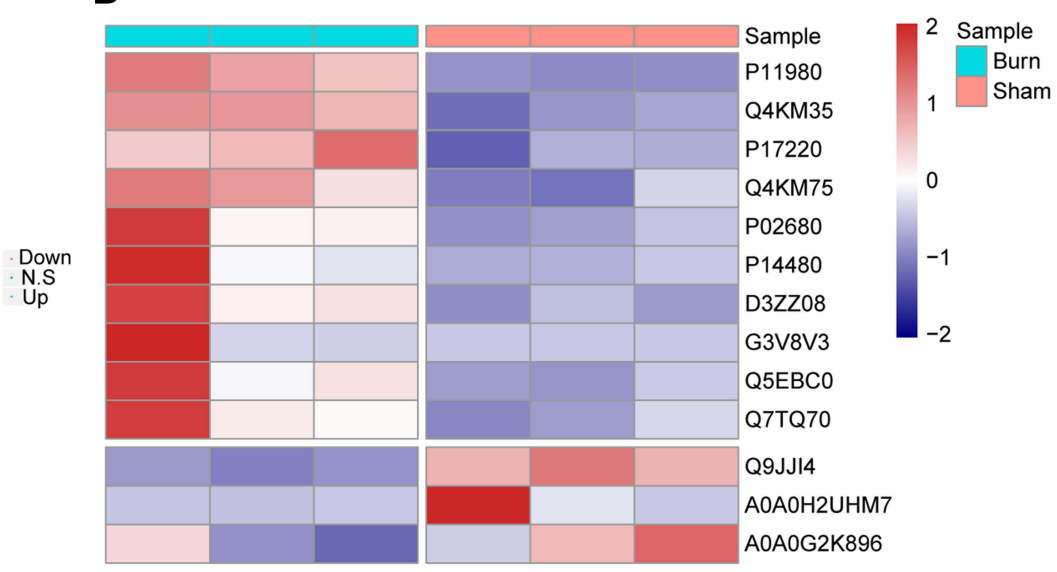

F

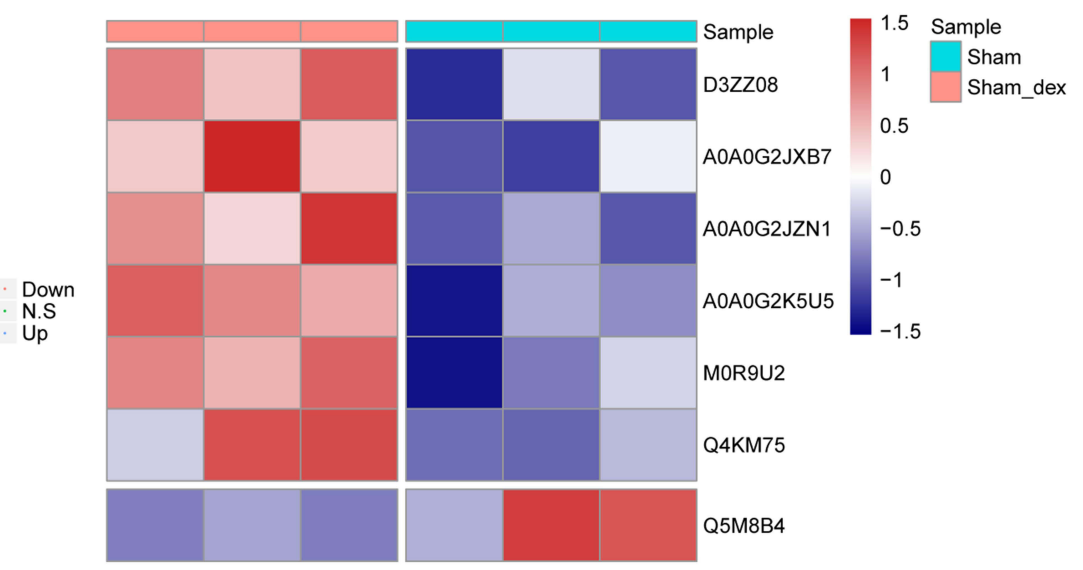

Figure 3 Differentially expressed protein analysis results. (A) The differentially expressed protein results of Burn-Dex vs Burn were displayed in volcano graph. The horizontal axis represents the differential expression multiples (Log2FC), and the vertical axis is - log 10 (FDR). The blue dots are upregulated proteins, the red dots are downregulated proteins and the green dots are not significantly regulated proteins. (B) The differentially expressed protein results of Burn-Dex vs Burn were displayed in heat map. The horizontal axis represents the sample and the vertical axis represents different proteins. Red indicates high protein expression, and blue indicates low protein expression. (C and $\mathbf{D})$ The differentially expressed protein results of Burn vs Sham were displayed in volcano graph and heat map. (E and $\mathbf{F})$ The differentially expressed protein results of Sham-Dex vs Sham were displayed in volcano graph and heat map. 
Table I The Differentially Expressed Proteins Were Significantly Enriched in the Following Three GO Terms

\begin{tabular}{|l|l|l|l|}
\hline ID & Ontology & GO.Term.Description & FDR \\
\hline GO:0051603 & BP & Proteolysis involved in cellular protein catabolic process & $<0.0$ I \\
GO:0005839 & CC & Proteasome core complex & $<0.0$ I \\
GO:0004298 & MF & Threonine-type endopeptidase activity & $<0.0$ I \\
\hline
\end{tabular}

Table 2 The Top 10 Significantly Enriched Pathways

\begin{tabular}{|c|c|c|c|}
\hline ID & Ontology & Pathway Description & FDR \\
\hline rno03050 & KEGG & Proteasome & 0.000013 \\
\hline $\begin{array}{l}\text { RNO- } \\
\text { II6909I }\end{array}$ & RCTM & Activation of NF-kappaB in B cells & 0.00096 \\
\hline $\begin{array}{l}\text { RNO- } \\
1234176\end{array}$ & RCTM & Oxygen-dependent proline hydroxylation of Hypoxia-inducible Factor Alpha & 0.00096 \\
\hline $\begin{array}{l}\text { RNO- } \\
\text { I236978 }\end{array}$ & RCTM & Cross-presentation of soluble exogenous antigens (endosomes) & 0.00096 \\
\hline $\begin{array}{l}\text { RNO- } \\
\text { I74084 }\end{array}$ & RCTM & Autodegradation of CdhI by Cdhl:APC/C & 0.00096 \\
\hline $\begin{array}{l}\text { RNO- } \\
174113\end{array}$ & RCTM & SCF-beta-TrCP mediated degradation of Emil & 0.00096 \\
\hline $\begin{array}{l}\text { RNO- } \\
174 \mid 54\end{array}$ & RCTM & APC/C:Cdc20 mediated degradation of Securin & 0.00096 \\
\hline $\begin{array}{l}\text { RNO- } \\
174178\end{array}$ & RCTM & $\begin{array}{l}\text { APC/C:Cdh I mediated degradation of Cdc20 and other APC/C:CdhI targeted proteins in late mitosis/ } \\
\text { early GI }\end{array}$ & 0.00096 \\
\hline $\begin{array}{l}\text { RNO- } \\
174184\end{array}$ & RCTM & Cdc20:Phospho-APC/C mediated degradation of Cyclin A & 0.00096 \\
\hline $\begin{array}{l}\text { RNO- } \\
176408\end{array}$ & RCTM & Regulation of APC/C activators between GI/S and early anaphase & 0.00096 \\
\hline
\end{tabular}

usually used to show changes about intestinal permeability. ${ }^{37}$ In this study, we have successfully constructed an intestinal barrier dysfunction rats model through burns. The plasma DAO and plasma FITCdextran in the Burn group decreased significantly after Dex administration, indicating that Dex could reduce the intestinal permeability. Our data were in line with a similar previous research, which demonstrated that Dex could attenuate intestinal microcirculatory dysfunction to protect the intestinal barrier. ${ }^{19}$ In addition, our results supplemented another aspect that Dex could also regulate the permeability of the intestine to protect the intestinal barrier.
Besides the physiological indicators, we also performed proteomics analysis in order to further understand the underlying mechanism of the Dex action in intestinal barrier repair. Then, we found four target proteins, Q4KM35, Q9JHW0, A0A0G2K896 and A0A0H2UHM7, which meet all our conditions below: a.) differentially express in Burn-Dex and Burn, b.) differentially express in Burn and Sham, c.) do not differentially express in Sham-Dex and Sham, which corresponding to the genes of Psmb10, Psmb7, RGD1310507 and LOC100909441. Until now, no studies to our knowledge have focused on the roles of these 4 genes in intestinal barrier repair. 
In order to further understand the possible mechanism behind Dex administration in intestinal barrier repair, we conducted an enrichment analysis. We found that RGD1310507 and LOC100909441 were not significantly enriched in any terms and pathways in GO and KEGG analysis, while Psmb7 and Psmb10 were significantly enriched in GO and KEGG analysis. The results showed that, after Dex administration, the differentially expressed genes were mainly enriched in the pathways related to the proteasome. Psmb10 and Psmb7 are significantly enriched in the three GO terms: proteolysis involved in cellular protein catabolic process, proteasome core complex and threonine-type endopeptidase activity. They are also significantly enriched in 49 pathways such as proteasome, activation of NF-kappaB in B cells and so on. Thus, we pay more attention to Psmb10 and Psmb7.

Proteasome is a key regulator of inflammation. ${ }^{38}$ Under normal circumstances, the expression of the proteasome is generally low and can be induced under certain circumstances, ${ }^{38}$ and the dysfunction of the proteasome will cause various human diseases. ${ }^{39}$ Regarding Psmb10 and Psmb7, they belong to Proteasome $\beta$ subunits (PSMB) family. PSMB family, a component of the ubiquitinproteasome system, has been documented to play an important role in inflammation, tumors and degenerative diseases. ${ }^{40,41}$

At present, there is almost no relevant research on Psmb10 and Psmb7 in the intestinal barrier, but in other diseases, ${ }^{40-44}$ there are researches demonstrated that high expression is often associated with negative clinical conditions. It has been reported that high PSMB7 expression is an unfavourable prognostic marker in breast cancer. ${ }^{40}$ And it has been evidenced that the inhibition of PSMB10 could reduce the Angiotensin II-induced atrial fibrillation. ${ }^{41}$ Moreover, Ghouzali et al's research has reported that intestinal hyperpermeability was blunted by intraperitoneal injection of selective proteasome inhibitor in rats. ${ }^{39}$ In addition, other studies suggest that proteasome inhibition prevented colitis in mice. ${ }^{43,45,46}$ As we all know, intestinal permeability is mainly regulated by a complex protein system that constitutes tight junctions (TJ). ${ }^{39}$ The decrease of tight junction proteins will lead to intestinal barrier disruption and hyperpermeability. ${ }^{3}$ Previous experimental data indicated that down-regulation of occludin protein (an important member of tight junction) expression may be related to its increased degradation by the proteasome system. ${ }^{47}$ Thus, based on the previous studies, we speculate that Dex may inhibit the activation of proteasome by reducing the expression of Psmb10 and Psmb7 to maintain the intestinal barrier.

\section{Conclusions}

In conclusion, we successfully constructed a burn-rat model and identified two possible protein targets of Dex in intestinal barrier repair, which are Q4KM35 and Q9JHW0 corresponding to genes Psmb10 and Psmb7, respectively. This will contribute to the better application of Dex in the repair of intestinal barrier dysfunction caused by burns. In the future, we will also further explore the functions of these proteins and genes to broaden our understanding of action of Dex.

\section{Data Sharing Statement}

The datasets used and analysed in the present research are available from the corresponding author on reasonable request.

\section{Ethics Approval and Consent to Participate}

Our study has been approved by the Animal Ethical and Welfare of Tianjin Medical University (Tianjin, China) in accordance with the Institutional Guidelines (SYXK (Tianjin), 2019-0004).

\section{Acknowledgments}

We thank HPS Gene Technology Co., Ltd. for their excellent support in the article.

\section{Author Contributions}

Chao Qin, Yi Jiang and Mingdong Yu should be considered joint first authors. All authors made a significant contribution to the work reported, whether that is in the conception, study design, execution, acquisition of data, analysis and interpretation, or in all these areas; took part in drafting, revising or critically reviewing the article; gave final approval of the version to be published; have agreed on the journal to which the article has been submitted; and agree to be accountable for all aspects of the work. Consent for publication: all authors have read and agreed to the published version of the manuscript.

\section{Funding}

This work was supported by the Chinese National Natural Science Foundation [Grant NO. 81671888 and 81772043] 
and the Natural Science Foundation of Tianjin [Grant NO. 18JCQNJC13000, Chao Qin]. This work was supported by the State Key Laboratory of Medicinal Chemical Biology [No. 2018101].

\section{Disclosure}

The authors declare that there is no conflict of interest regarding the publication of this article.

\section{References}

1. Gross-Amat O, Guillen M, Salmon D, Nataf S, Auxenfans C. Characterization of a topically testable model of burn injury on human skin explants. Int J Mol Sci. 2020;21(18):6956. doi:10.3390/ ijms 21186956

2. He S, Alonge O, Agrawal P, et al. Epidemiology of burns in rural Bangladesh: an update. Int J Environ Res Public Health. 2017;14 (4):381. doi:10.3390/ijerph14040381

3. He W, Wang Y, Wang P, Wang F. Intestinal barrier dysfunction in severe burn injury. Burns Trauma. 2019;7:24. doi:10.1186/s41038019-0162-3

4. Wang P, Feng YH, Wang SB, Wang Y, Huang YL, Wang FJ. [Significance of intestinal fatty acid binding protein in evaluation of intestinal barrier dysfunction of mice at early stage of severe burn injury]. Zhonghua Shao Shang Za Zhi. 2019;35(6):459-463. Chinese. doi:10.3760/cma.j.issn.1009-2587.2019.06.012

5. Yang $\mathrm{X}$, Chen J, Bai H, et al. Inhibition of $\mathrm{Na}+/ \mathrm{H}+$ exchanger 1 by cariporide reduces burn-induced intestinal barrier breakdown. Burns. 2013;39(8):1557-1564. doi:10.1016/j.burns.2013.04.007

6. Song Y, Li Y, Xiao Y, et al. Neutralization of interleukin-17A alleviates burn-induced intestinal barrier disruption via reducing pro-inflammatory cytokines in a mouse model. Burns Trauma. 2019;7:37. doi:10.1186/s41038-019-0177-9

7. Kraehenbuhl JP, Pringault E, Neutra MR. Review article: intestinal epithelia and barrier functions. Aliment Pharmacol Ther. 1997;11 (Suppl 3):3-9. doi:10.1111/j.1365-2036.1997.tb00803.x

8. Vancamelbeke M, Vermeire S. The intestinal barrier: a fundamental role in health and disease. Expert Rev Gastroenterol Hepatol. 2017;11(9):821-834. doi:10.1080/17474124.2017.1343143

9. Branca JJV, Gulisano M, Nicoletti C. Intestinal epithelial barrier functions in ageing. Ageing Res Rev. 2019;54:100938. doi:10.1016/ j.arr.2019.100938

10. Al-Ghoul WM, Khan M, Fazal N, Sayeed MM. Mechanisms of postburn intestinal barrier dysfunction in the rat: roles of epithelial cell renewal, E-cadherin, and neutrophil extravasation. Crit Care Med. 2004;32(8):1730-1739. doi:10.1097/01.CCM. 0000132896.62368 .01

11. Feng Y, Huang Y, Wang Y, Wang P, Wang F. Severe burn injury alters intestinal microbiota composition and impairs intestinal barrier in mice. Burns Trauma. 2019;7:20. doi:10.1186/s41038-019-0156-1

12. Asmussen S, Maybauer DM, Fraser JF, Jennings K, George S, Maybauer MO. A meta-analysis of analgesic and sedative effects of dexmedetomidine in burn patients. Burns. 2013;39(4):625-631. doi:10.1016/j.burns.2013.01.008

13. Lee S. Dexmedetomidine: present and future directions. Korean $J$ Anesthesiol. 2019;72(4):323-330. doi:10.4097/kja.19259

14. Skrobik Y, Duprey MS, Hill NS, Devlin JW. Low-dose nocturnal dexmedetomidine prevents ICU delirium. A randomized, placebo-controlled trial. Am J Respir Crit Care Med. 2018;197 (9):1147-1156. doi:10.1164/rccm.201710-1995OC

15. Moc C, Taylor AE, Chesini GP, et al. Physiological activation of Akt by PHLPP1 deletion protects against pathological hypertrophy. Cardiovasc Res. 2015;105(2):160-170. doi:10.1093/cvr/cvu243
16. Mei B, Li J, Zuo Z. Dexmedetomidine attenuates sepsis-associated inflammation and encephalopathy via central alpha2A adrenoceptor. Brain Behav Immun. 2021;91:296-314. doi:10.1016/j. bbi.2020.10.008

17. Geze S, Cekic B, Imamoglu M, et al. Use of dexmedetomidine to prevent pulmonary injury after pneumoperitoneum in ventilated rats. Surg Laparosc Endosc Percutan Tech. 2012;22(5):447-453. doi:10.1097/SLE.0b013e31826183df

18. Kaye AD, Chernobylsky DJ, Thakur P, et al. Dexmedetomidine in Enhanced Recovery After Surgery (ERAS) protocols for postoperative pain. Curr Pain Headache Rep. 2020;24(5):21. doi:10.1007/ s11916-020-00853-z

19. Yeh YC, Wu CY, Cheng YJ, et al. Effects of dexmedetomidine on intestinal microcirculation and intestinal epithelial barrier in endotoxemic rats. Anesthesiology. 2016;125(2):355-367. doi:10.1097/ ALN.0000000000001135

20. Qin C, Jiang Y, Chen X, et al. Dexmedetomidine protects against burn-induced intestinal barrier injury via the MLCK/p-MLC signalling pathway. Burns. 2021. doi:10.1016/j.burns.2021.01.017

21. Sun Y, Gao Q, Wu N, Li SD, Yao JX, Fan WJ. Protective effects of dexmedetomidine on intestinal ischemia-reperfusion injury. Exp Ther Med. 2015;10(2):647-652. doi:10.3892/etm.201 5.2561

22. Zhang XY, Liu ZM, Wen SH, et al. Dexmedetomidine administration before, but not after, ischemia attenuates intestinal injury induced by intestinal ischemia-reperfusion in rats. Anesthesiology. 2012;116 (5):1035-1046. doi:10.1097/ALN.0b013e3182503964

23. Serafini S, Santos MM, Aoun Tannuri AC, et al. Is hematoxylin-eosin staining in rectal mucosal and submucosal biopsies still useful for the diagnosis of Hirschsprung disease? Diagn Pathol. 2017;12(1):84. doi:10.1186/s13000-017-0673-9

24. Chiu CJ, McArdle AH, Brown R, Scott HJ, Gurd FN. Intestinal mucosal lesion in low-flow states. I. A morphological, hemodynamic, and metabolic reappraisal. Arch Surg. 1970;101(4):478-483. doi:10.1001/archsurg.1970.01340280030009

25. Wisniewski JR, Zougman A, Nagaraj N, Mann M. Universal sample preparation method for proteome analysis. Nat Methods. 2009;6 (5):359-362. doi:10.1038/nmeth.1322

26. Ritchie ME, Phipson B, Wu D, et al. Limma powers differential expression analyses for RNA-sequencing and microarray studies. Nucleic Acids Res. 2015;43(7):e47. doi:10.1093/nar/gkv007

27. Szklarczyk D, Gable AL, Lyon D, et al. STRING v11: protein-protein association networks with increased coverage, supporting functional discovery in genome-wide experimental datasets. Nucleic Acids Res. 2019;47(D1):D607-D13. doi:10.1093/nar/gky1131

28. Peterson CY, Costantini TW, Loomis WH, et al. Toll-like receptor-4 mediates intestinal barrier breakdown after thermal injury. Surg Infect (Larchmt). 2010;11(2):137-144. doi:10.1089/sur.2009.053

29. Mahmoud M, Barbi E, Mason KP. Dexmedetomidine: what's new for pediatrics? A narrative review. J Clin Med. 2020;9(9):2724. doi:10.3390/jcm9092724

30. Van Spaendonk H, Ceuleers H, Witters L, et al. Regulation of intestinal permeability: the role of proteases. World J Gastroenterol. 2017;23(12):2106-2123. doi:10.3748/wjg.v23.i12.2106

31. Odenwald MA, Turner JR. Intestinal permeability defects: is it time to treat? Clin Gastroenterol Hepatol. 2013;11(9):1075-1083. doi:10.1016/j.cgh.2013.07.001

32. Schoultz I, Keita AV. The intestinal barrier and current techniques for the assessment of gut permeability. Cells. 2020;9(8):1909. doi:10.3390/cells9081909

33. Meng Y, Zhang Y, Liu M, et al. Evaluating intestinal permeability by measuring plasma endotoxin and diamine oxidase in children with acute lymphoblastic leukemia treated with high-dose methotrexate. Anticancer Agents Med Chem. 2016;16(3):387-392. doi:10.2174/ 1871520615666150812125955 
34. $\mathrm{Li} \mathrm{BR}, \mathrm{Wu} \mathrm{J}, \mathrm{Li} \mathrm{HS}$, et al. In vitro and in vivo approaches to determine intestinal epithelial cell permeability. $J$ Vis Exp. 2018;140. doi:10.3791/57032

35. Honzawa Y, Nakase H, Matsuura M, Chiba T. Clinical significance of serum diamine oxidase activity in inflammatory bowel disease: importance of evaluation of small intestinal permeability. Inflamm Bowel Dis. 2011;17(2):E23-5. doi:10.1002/ibd.21588

36. Wang Y, An Y, Ma W, et al. 27-hydroxycholesterol contributes to cognitive deficits in APP/PS1 transgenic mice through microbiota dysbiosis and intestinal barrier dysfunction. $J$ Neuroinflammation. 2020;17(1):199. doi:10.1186/s12974-020-01873-7

37. Guo Y, Li H, Liu Z, et al. Impaired intestinal barrier function in a mouse model of hyperuricemia. Mol Med Rep. 2019;20 (4):3292-3300. doi:10.3892/mmr.2019.10586

38. Rousseau A, Bertolotti A. Regulation of proteasome assembly and activity in health and disease. Nat Rev Mol Cell Biol. 2018;19 (11):697-712. doi:10.1038/s41580-018-0040-z

39. Ghouzali I, Lemaitre C, Bahlouli W, et al. Targeting immunoproteasome and glutamine supplementation prevent intestinal hyperpermeability. Biochim Biophys Acta Gen Subj. 2017;1861 (1):3278-3288. doi:10.1016/j.bbagen.2016.08.010

40. Wang CY, Li CY, Hsu HP, et al. PSMB5 plays a dual role in cancer development and immunosuppression. Am J Cancer Res. 2017;7 (11):2103-2120.

41. Li J, Wang S, Bai J, et al. Novel role for the immunoproteasome subunit PSMB10 in angiotensin II-induced atrial fibrillation in mice. Hypertension. 2018;71(5):866-876. doi:10.1161/HYPERTENSION AHA.117.10390
42. Kyrychenko VO, Nagibin VS, Tumanovska LV, et al. Knockdown of PSMB7 induces autophagy in cardiomyocyte cultures: possible role in endoplasmic reticulum stress. Pathobiology. 2014;81(1):8-14. doi: $10.1159 / 000350704$

43. Schmidt N, Gonzalez E, Visekruna A, et al. Targeting the proteasome: partial inhibition of the proteasome by bortezomib or deletion of the immunosubunit LMP7 attenuates experimental colitis. Gut. 2010;59(7):896-906. doi:10.1136/gut.2009.203554

44. Waku T, Katayama H, Hiraoka M, et al. NFE2L1 and NFE2L3 complementarily maintain basal proteasome activity in cancer cells through CPEB3-mediated translational repression. Mol Cell Biol. 2020;40(14). doi:10.1128/MCB.00010-20.

45. Basler M, Dajee M, Moll C, Groettrup M, Kirk CJ. Prevention of experimental colitis by a selective inhibitor of the immunoproteasome. J Immunol. 2010;185(1):634-641. doi:10.4049/ jimmunol.0903182

46. Inoue $S$, Nakase $H$, Matsuura $M$, et al. The effect of proteasome inhibitor MG132 on experimental inflammatory bowel disease. Clin Exp Immunol. 2009;156(1):172-182. doi:10.1111/j.13652249.2008.03872.x

47. Coeffier M, Gloro R, Boukhettala $\mathrm{N}$, et al. Increased proteasome-mediated degradation of occludin in irritable bowel syndrome. $A m \quad J$ Gastroenterol. 2010;105(5):1181-1188. doi:10.1038/ajg.2009.700
Journal of Inflammation Research

\section{Publish your work in this journal}

The Journal of Inflammation Research is an international, peerreviewed open-access journal that welcomes laboratory and clinical findings on the molecular basis, cell biology and pharmacology of inflammation including original research, reviews, symposium reports, hypothesis formation and commentaries on: acute/chronic inflammation; mediators of inflammation; cellular processes; molecular

\section{Dovepress}

mechanisms; pharmacology and novel anti-inflammatory drugs; clinical conditions involving inflammation. The manuscript management system is completely online and includes a very quick and fair peerreview system. Visit http://www.dovepress.com/testimonials.php to read real quotes from published authors. 